\title{
Clinicopathological Evaluation of 15 Ectopic Adrenal Tissues
}

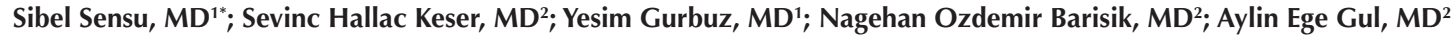 \\ 'Department of Pathology, Medical Faculty, Istinye University, Istanbul, Turkey \\ 2Department of Pathology, Dr. Lutfi Kirdar Kartal Training and Research Hospital, University of Health Sciences, Istanbul, Turkey
}

\begin{abstract}
Background: Ectopic adrenal tissue is a rare condition associated with embryological development defects seen in many different areas in the abdomen and pelvis. Here, we aimed to discuss the clinicopathological features of ectopic adrenal tissues diagnosed in our clinic, in light of the literature.

Methods: We included cases of ectopic adrenal tissues incidentally detected in the specimens from patients undergoing operation for various diagnoses during 2012- March 2020. The cases were examined according to gender, age, location and accompanying pathological diagnoses. During this period, 15 cases of ectopic adrenal tissues (6 paratubal, 3 paraovarian, 2 paratesticular, 1 spermatic cord, 1 paraaortic, 1 liver capsule, 1 omentum) were detected accompanied by two endometrial carcinomas, two serous cystadenomas, one seminoma, one mixed germ cell tumor, one bilateral ovarian serous carcinoma and hepatic high-grade colon adenocarcinoma metastasis.

Results: In this report, the fifth ectopic adrenal tissue accompanying a malignant testicular tumor, the fifth and sixth ectopic adrenal tissues occurring with ovarian serous cystadenoma, the first case observed with serous cystadenocarcinoma and the first case detected with hepatic adenocarcinoma metastasis are presented. Our cases are mostly women and adult men.

Conclusion: Ectopic adrenal tissues can lead to hormonal problems and also to adrenal cortex and medulla neoplasms. Microscopically, they may be confused with clear cell gynecological and germ cell tumors. If the ectopic focus is misdiagnosed as an implant, a benign entity may be incorrectly reported as malignant. Also, it is important to prevent mis-staging in malignancies. For precise diagnosis, an immunopanel such as inhibin, melan A, and calretinin can be performed.

Keywords: Clear cell tumors, Ectopic adrenal, Ovarian tumor, Testicular neoplasms

Cite this article as: Sensu S, Keser SH, Gurbuz Y, Barisik NO, Gul AE. Clinicopathological evaluation of 15 ectopic adrenal tissues. Arch Iran Med. 2021;24(4):301-305. doi: 10.34172/aim.2021.42
\end{abstract}

Received: April 17, 2020, Accepted: July 22, 2020, ePublished: April 1, 2021

\section{Introduction}

Ectopic adrenal tissue, first defined by Morgagni in 1740 , is the presence of adrenal gland tissue in different locations, with an incidence reported between $1 \%-9.3 \% .^{1-}$ ${ }^{3}$ It is detected in up to $50 \%$ of cases in newborns and early childhood, and is much rarer in adults $(1 \%) .{ }^{4}$ Although found in both sexes, it is more common in men. It is usually seen with an undescended testicle, inguinal hernia or hydrocele in children, and incidentally during a surgical procedure at a later age. ${ }^{1,2}$ Cases have been reported in the kidneys, retroperitoneal adipose tissue, ovaries, paratubal area, spermatic cord, testicles, pancreas, liver, stomach and transverse colon. ${ }^{3}$

Ectopic adrenal tissue is generally seen as a benign, hormonally inactive, millimetric yellow mass requiring no follow-up. ${ }^{1,2}$ However, it is considered clinically important because of the possibility of malignant transformation in heterotopic tissues, compensatory hyperplasia after adrenal removal, or adrenal insufficiency when heterotopic tissue is removed. ${ }^{1}$ In this study, the clinicopathological features of the ectopic adrenal tissues detected in our clinic were examined and discussed in light of the literature.

\section{Materials and Methods}

In this retrospective study, cases of ectopic adrenal tissues detected incidentally in specimens of patients operated for various reasons during 2012-March 2020 were analyzed. Regardless of age, hormonal status, clinical diagnosis and co-existing clinicopathological features, all the cases with a diagnosis of "ectopic adrenal tissue" in histopathological examination report were included in the study. Glass slides, paraffin blocks and patient reports belonging to the cases were retrieved from Kartal Dr Lutfi Kırdar City Hospital, Pathology Department archives. Exclusion criteria were lack of slides, paraffin blocks or patient reports. All slides stained with hematoxylin and eosin were re-examined and classified according to pre-defined parameters.

\section{Immunobistochemical Analysis}

Toverifytheadrenalcortextissuewithimmunohistochemical demonstration of inhibin, appropriate paraffin blocks were selected and four-micrometer thick sections were taken from each block up on poly-L-lysine-coated slides. Immunohistochemical examination was performed on deparaffinized sections using the standard avidin- 
biotin-peroxidase complex method with automated immunostainer (BenchMark ULTRA, Ventana medical system, Tucson, AZ, USA). Formalin-fixed paraffinembedded blocks were analyzed for expression of monoclonal antibody against the $\alpha$ subunit of inhibin (Inhibin alpha Novocastra-clone AMY82-1/100 dilution). Ovarian tissue was used as positive control. Cytoplasmic inhibin staining was considered as positive staining.

Gender, age, location and accompanying pathological findings were recorded. The PubMed and Medline Complete databases were scanned for articles in English published between 2000 and March 2020. The cases diagnosed in our clinic were presented and compared with literature data.

\section{Results}

There were 15 cases of ectopic adrenal tissue in this study and gender, age, location, size, surgical approach and accompanying pathological findings are presented in Table 1. The sizes of the cases were between 0.1 and $0.5 \mathrm{~cm}$. Eleven $(73 \%)$ of the patients were women and four $(27 \%)$ were men. Their age varied between 10 and 69 years (mean age, 46.0). The mean age was 29 years (10, 19, 27 and 60 years) for men and 52.2 years for women. Ectopic adrenal tissue was located on the left side in eight (53\%) and on the right side in five $(47 \%)$ patients. In women, $82 \%$ of the cases were in the paratubal ( 6 cases,
$55 \%$ ) and paraovarian (3 cases, 27\%) regions. Two cases were paratesticular (13\%), and the remaining were located in the spermatic cord, paraaortic region, liver capsule and omentum. Of 15 cases operated for various diagnoses, six (40\%) were accompanied by a malignant tumor and two (13\%) by an epithelioid benign tumor. Two cases (33\%) of paratubal location were operated on due to endometrial carcinoma, two (33\%) for leiomyoma, one (7\%) for serous cystadenoma and one for both serous cystadenoma and leiomyoma. One case detected in the spermatic cord had a seminoma. One patient with paratesticular location was operated on due to mixed germ cell tumor. The case detected in the paraaortic location was operated on due to bilateral ovarian serous carcinoma. Colon adenocarcinoma metastasis was observed in the patient with liver capsule location, and fat necrosis was observed in the patient with omentum location. The cases were hormonally inactive and no neoplastic development was observed in ectopic adrenal tissues.

On microscopic examination, zona glomerulosa cells with oval or round nuclei, amphophilic cytoplasm; zona fasciculata cells with abundant clear cytoplasm; and zona reticularis cells with eosinophilic cytoplasm were seen (Figures 1 and 2). On immunohistochemical examination, adrenal cortex cells showed cytoplasmic inhibin positivity (Figure 3). Cells belonging to the adrenal medulla were not seen in any of the cases.

Table 1. Gender, Age, Location, Size, Surgical Approach and Accompanying Pathological Characteristics of Ectopic Adrenal Tissue.

\begin{tabular}{|c|c|c|c|c|c|c|c|}
\hline \multirow{2}{*}{ Case \# } & \multicolumn{3}{|c|}{ Ectopic adrenal tissue } & \multirow{2}{*}{ Gender } & \multirow{2}{*}{ Age } & \multirow{2}{*}{ Surgical Approach } & \multirow{2}{*}{ Accompanying Pathological Finding } \\
\hline & Location & Side & $\operatorname{Size}(\mathrm{cm})$ & & & & \\
\hline 1 & Paratubal & Left & 0.2 & $\mathrm{~F}$ & 69 & Uterus, $\mathrm{TAH}+\mathrm{BSO}$ & Leiomyoma, serous cystadenoma \\
\hline 2 & Paratubal & Left & 0.3 & $\mathrm{~F}$ & 58 & Uterus, TAH+BSO & Endometrial adenocarcinoma, endometrioid type \\
\hline 3 & Paratubal & Right & 0.3 & $\mathrm{~F}$ & 52 & Uterus, $\mathrm{TAH}+\mathrm{BSO}$ & Leiomyoma \\
\hline 4 & Paratubal & Right & 0.2 & $\mathrm{~F}$ & 54 & Uterus, $\mathrm{TAH}+\mathrm{BSO}$ & Leiomyoma \\
\hline 5 & Paratubal & Left & 0.15 & $\mathrm{~F}$ & 58 & Uterus, $\mathrm{TAH}+\mathrm{BSO}$ & Left ovary, serous cystadenoma \\
\hline 6 & Paratubal & Right & 0.4 & $\mathrm{~F}$ & 57 & Uterus, $\mathrm{TAH}+\mathrm{BSO}$ & Endometrioid adenocarcinoma, Grade 2 \\
\hline 7 & Paraovarian & Right & 0.4 & $\mathrm{~F}$ & 48 & Uterus, $\mathrm{TAH}+\mathrm{BSO}$ & Leiomyoma \\
\hline 8 & Paraovarian & Left & 0.4 & $\mathrm{~F}$ & 42 & Right and left tubectomy & Paratubal cyst \\
\hline 9 & $\begin{array}{l}\text { Adipose tissue adjacent } \\
\text { to ovary and tuba } \\
\text { uterina }\end{array}$ & Left & 0.4 & $\mathrm{~F}$ & 39 & Uterus, $\mathrm{TAH}+\mathrm{BSO}$ & Endometriotic cyst \\
\hline 10 & Paratesticular & Left & 0.1 & M & 19 & $\begin{array}{l}\text { Radical inguinal } \\
\text { orchiectomy }\end{array}$ & Testicular mixed germ cell tumor \\
\hline 11 & Paratesticular & Left & 0.2 & M & 10 & Testicle, orchiectomy & $\begin{array}{l}\text { Calcified and atrophic testis tissue (undescended } \\
\text { testis) }\end{array}$ \\
\hline 12 & $\begin{array}{l}\text { Adjacent to spermatic } \\
\text { cord }\end{array}$ & left & 0.2 & M & 27 & Testicle, orchiectomy & Classic type seminoma \\
\hline 13 & $\begin{array}{l}\text { Paraaortic lymph nodes } \\
\text { near inferior mesenteric } \\
\text { artery }\end{array}$ & $?$ & 0.5 & $\mathrm{~F}$ & 57 & Uterus, $\mathrm{TAH}+\mathrm{BSO}$ & Right-left ovary, high grade serous carcinoma \\
\hline 14 & $\begin{array}{l}\text { Adjacent to liver } \\
\text { capsule }\end{array}$ & Right & 0.5 & M & 60 & Liver, right hepatectomy & Liver, adenocarcinoma metastasis \\
\hline 15 & Omentum & - & 0.1 & $\mathrm{~F}$ & 41 & $\begin{array}{l}\text { Uterus, } \mathrm{TAH}+\mathrm{BSO}+ \\
\text { omentectomy }\end{array}$ & $\begin{array}{l}\text { Outer wall of uterus, fat necrosis, mixed } \\
\text { inflammatory infiltration } \\
\text { Right ovary, mixed inflammatory infiltration }\end{array}$ \\
\hline
\end{tabular}

F, Female; M, Male; TAH+BSO, Total abdominal hysterectomy+bilateral salpingoopherectomy. 


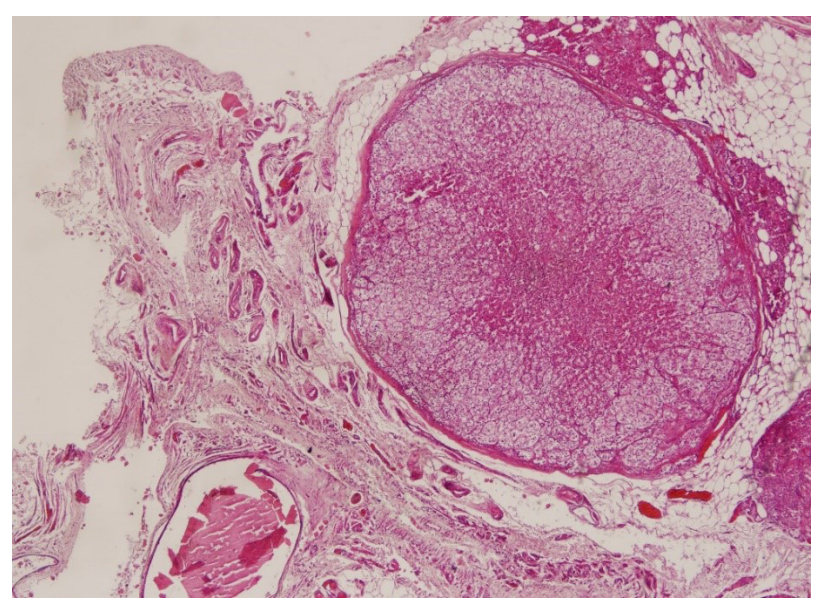

Figure 1. Ectopic Adrenal Tissue in the Right Paratubal Region of a Patient Operated on for Endometrial Cancer (H\&E, X4).

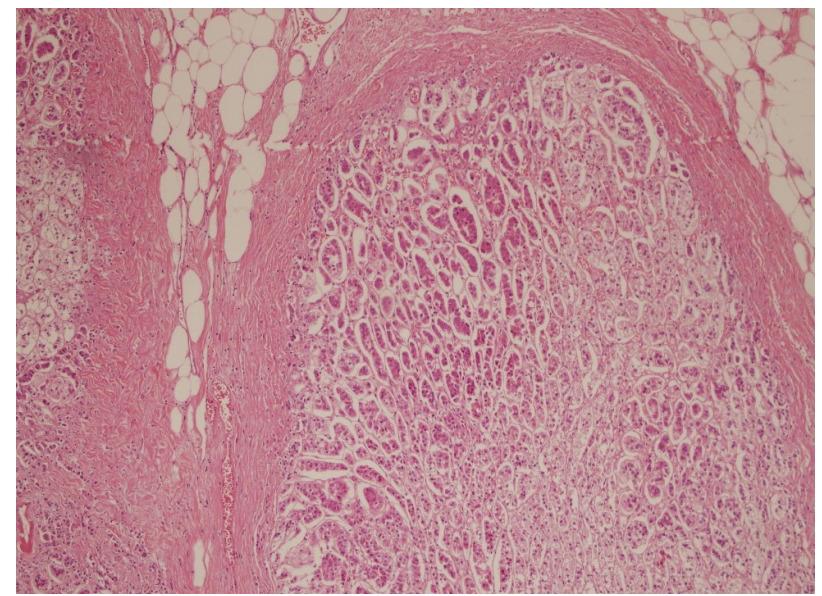

Figure 2. Ectopic Adrenal Tissue Found Adjacent to the Liver Capsule of a Patient Operated on for Liver Metastasis (H\&E, X10).

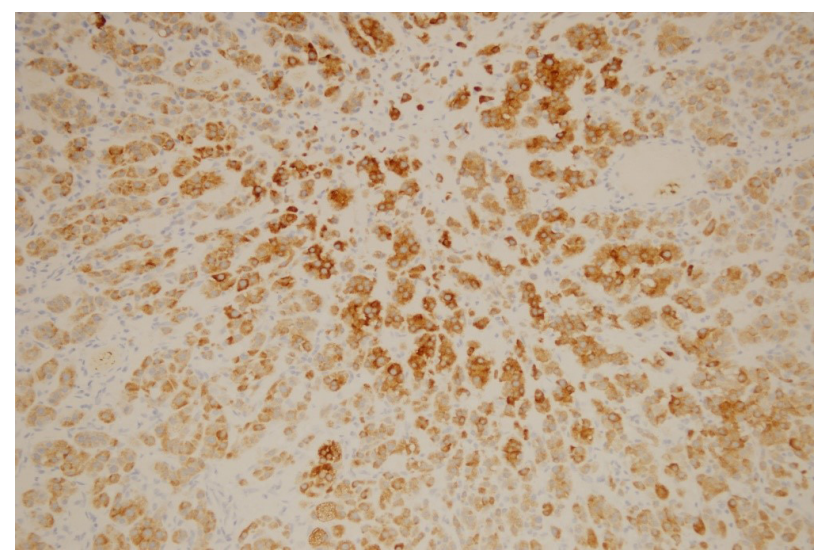

Figure 3. Cytoplasmic Inhibin Expression in Ectopic Adrenal Cortex Cells of a Patient Operated on for Serous Carcinoma (Inhibin. X20).

\section{Discussion}

Adrenal gland has a dual embryological origin. The cortex develops from the coelomic epithelium of the urogenital ridge, and the medulla from the neural crest cells. Since the testicles and adrenal cortex develop from the same urogenital ridge, adrenal tissue can migrate along with the descending gonads in the early embryonic period. Although ectopic tissue is mostly atrophied, it sometimes remains as an adrenal rest with a dimension usually less than $1 \mathrm{~cm} .{ }^{1}$ Accordingly, all our cases were between 0.1 to $0.5 \mathrm{~cm}$ in size, most of which too small to be detected with naked eye.

Although both cortex and medulla tissues can be seen microscopically in ectopic adrenal tissues, often only adrenocortical type cells are found. Similarly, we observed cells related to all three layers of the cortex; however, no medullar tissue was found.

\section{Cases in Men}

Ectopic adrenal tissue is reported more frequently in children and in men. Mares et al reported ectopic adrenal tissue in $3.8 \%$ of children who underwent surgery due to inguinoscrotal problems and $9.3 \%$ of those who had undescended testis operations 5 . Sullivan et al detected ectopic adrenal tissue in $2.7 \%$ of 935 groin surgeries performed in patients under the age of $16 .^{6}$ Ectopic adrenal tissue was reported near testes in $7.5 \%-15 \%$ of newborns. ${ }^{7}$ However, it was less common in adults. In a systematic review, publications mentioning ectopic adrenal tissue in the groin region of adults (inguinal ligament, intratesticular, spermatic cord, hernia sac) were screened; in 15 articles, ectopic adrenal (only cortical) tissue was detected in 21 male adults (mean age 43.5), and it was mostly found in hernia sacs $(47.6 \%) .{ }^{6}$ Contrarily, in our series of 15 cases, most of the cases were women; there was only four male patients and they were slightly younger than the women.

In the literature, few cases of adrenal ectopia along with testicular neoplasm have been reported. In three of them (43, 45 and 38 years of age), the accompanying neoplasm was seminoma and ectopic adrenal was incidentally detected in the spermatic cord after orchiectomy. ${ }^{2,8,9}$ In our series, one of the four male patients had seminoma, one had mixed germ cell tumor and one had adenocarcinoma metastasis to the liver. With our cases, the number of ectopic adrenals accompanying a malignant testicular tumor in the literature has increased to five. Of note, there is a case in the literature, a 3-month-old baby with teratoma in the testicle with an ectopic adrenal, detected in the spermatic cord. ${ }^{4}$

The cases associated with undescended testis and inguinal hernia are relatively more common. In a series of 21 adult patients, right or left inguinal hernia was reported in 13 cases $(62 \%)$ and undescended testes in four cases $(19 \%) .{ }^{7}$ We presented an ectopic adrenal in a 10 -year-old patient with an undescended testis.

Lastly, ectopic tissue accompanying malignant or benign entities in men can be asymptomatic, or lead to some consequences related with hormone secretion. Moreover, azoospermia and subsequently, infertility may occur due to obstruction of seminiferous tubules. ${ }^{1}$

In summary, ectopic adrenal tissues observed in men are frequently seen in undescended testis specimens, and 
sometimes in resections due to germ cell tumors of testis. Here, two of our cases were with germ cell tumor and one, with undescended testis. It is well known that, in undescended testes, germ cell tumors are common. Germ cell tumors can have various patterns; seminoma which is the most frequent germ cell tumor is a clear cell tumor; thus, ectopic adrenal should be kept in mind in order to prevent an error in staging of existing malignancy. To prove that such a focus is adrenal cortex, markers such as inhibin, melan A, calretinin; and to prove that it is not a germ cell tumor, PLAP, beta HCG, AFP may be helpful.

\section{Cases in Women}

In the literature, the most common location in women has been reported as ligamentum latum and mesosalpinx. ${ }^{10,11}$ Most of the cases in our series were also in this location.

Ectopic adrenal cases can be detected in operations performed due to benign and malignant ovarian neoplasms. In a case report, ectopic adrenal tissue with granulosa cell ovarian tumor was presented in a 50-yearold patient. $^{10}$ In another article, paratubal ectopic adrenal tissue was reported in a 26-year-old woman with mucinous cystadenoma. ${ }^{11}$ Additionally, there are four serous cystadenoma cases containing ectopic adrenal tissues (21-65 years of age). ${ }^{10,12-14}$ In our series, two female patients had ectopic adrenal in the paratubal area with serous cystadenoma and these constitute the 5th and 6th cases in the literature. Besides, one patient in our series had ectopic adrenal tissue accompanied with serous cystadenocarcinoma, and this is the first case in the literature.

Among our cases, two ectopic adrenal cases were associated with endometrial adenocarcinoma. In addition, ectopic adrenal tissues were detected incidentally in three cases operated on for leiomyoma. There are no reports in the literature regarding ectopic cases with uterine pathology. We had three cases (endometriotic cyst, paratubal cyst and adnexal/periadnexal inflammation) that accompanied ectopic adrenal tissues.

Hysterectomy is a frequent operation and, as seen in our series, ectopic adrenal tissue might be incidentally found in hysterectomy materials. In our series, ectopic adrenal tissues were observed to accompanying two endometrial and one ovarian carcinoma. Since both endometrial and ovarian gynecological malignancies have clear cell variants, differential diagnosis is required for the correct staging. Also, misinterpretation of ectopic adrenal tissue as an implant may lead to misdiagnosis of a clear cell adenoma as malignant. An immunopanel including inhibin, melan A, calretinin and related markers may be helpful to differentiate an ectopic adrenocortical tissue from a malignancy.

In the literature, a 23-year-old woman was described with high levels of serum testosterone and plasma adrenocorticotropic hormone with multiple retroperitoneal masses. ${ }^{15}$ As in this example, adrenal hyperplasia may develop as a result of ectopic adrenal masses. In another case, pheochromocytoma arising from ectopic adrenal tissue was presented in a 21 -year-old woman with multiple endocrine neoplasia type $2 \mathrm{~A}$ showing that, not only the cortex but also the medulla can be found in ectopic adrenal tissue, even leading to a neoplasia. ${ }^{16}$ According to data, functional hypertrophy may develop in ectopic adrenal tissue after removal or destruction of normal adrenal glands. ${ }^{1}$ Also, cases of Cushing's syndrome or primary aldosteronism arising from ectopic tissue located in the appendix may mimic carcinoid tumor. ${ }^{1}$ However, our cases were found incidentally, and therefore, we do not have any data on the levels of adrenal hormones.

\section{Ectopic Adrenal Tissue with Liver and Kidney Location}

For the first time in the literature, we present an ectopic adrenal tissue accompanying an adenocarcinoma metastasis in the liver. In such cases, adrenal insufficiency may develop after tumor resection since literature search reveals a patient who died after resection. ${ }^{1}$ Another problem may be differential diagnosis in some cases. In a case report of a 50-year-old woman with a liver mass, the authors warned that adrenal rest tumors in segment 7 may be misdiagnosed as lipid rich hepatocellular carcinoma and immunohistochemical panel containing CD56 and arginase-1 might be useful in differential diagnosis in those cases. ${ }^{17}$

In our case with hepatic location, a resection was performed due to liver metastasis and colon adenocarcinoma was detected incidentally. Since the case showed typical histology of colon carcinoma, the differential diagnosis was not problematic. However, in a metastasis with clear cells, the differential diagnosis might be difficult.

In a series of nine cases (five women and four men, aged 35-37 years), ectopic adrenal cases with intrarenal location were presented. Eight of the ectopic adrenal tissues were in the upper pole, one in the middle region and all contained only cortical tissue. Ectopic tissues were in the form of plaque-like, wedge-shaped, spherical or irregular nests. ${ }^{18}$ Findings favoring ectopic adrenal tissue were adrenal architecture, cell differentiation, absence of cytological atypia, necrosis or lymphovascular invasion, and cytoplasm containing clear lipid droplets in zona fasciculata-like cells. Also, adrenal cortical tissue is positive for inhibin and melan A, but negative for CD10, EMA and pancytokeratin. ${ }^{18}$

Of note, in the literature, there are cases with hormonally active ectopic adrenal tissues; a 28-year-old woman with virilization was presented who had a myelolipoma derived from ectopic adrenal tissue located in the left renal hilus ${ }^{19}$ and there are cases of ectopic aldosteronoma. ${ }^{20}$

In conclusion, together with our cases, the number of ectopic adrenal tissues accompanying a malignant testicular 
tumor has increased to five. In our series, two ectopic adrenal tissues were detected with serous cystadenoma and these are the 5 th and 6th cases in the literature. One patient had ectopic adrenal tissue accompanied by serous cystadenocarcinoma which is the first case in the literature. In addition, one case was found with adenocarcinoma metastasis to the liver for the first time. Based on our cases, ectopic adrenal tissues are not common only in boys but occur incidentally in urogenital and gastrointestinal operations performed for various indications in adult men and women. They can be either asymptomatic or cause hormonal pathologies of adrenal cortex and medulla. They can also lead to benign and malignant neoplasms. Those that compress the spermatic cord may cause azoospermia and infertility. Ectopic adrenal tissues may be histopathologically confused with clear cell types of some gynecological and germ cell tumors. Besides, if the ectopic focus is mis-evaluated as an implant, benign epithelial tumors may be mistakenly diagnosed as malignant or staging may be inaccurate in malignancies. A panel based on inhibin, melan A, calretinin and other required markers might be valuable to distinguish the adrenal cortex.

\section{Authors' Contribution}

SHK, NOB, AEG, YG, and SS: Conception or design of the work. SHK, NOB, and AEG: Data collection. SHK, NOB, AEG, YG, and SS: Data analysis and interpretation. SS, SHK, YG, AEG, and NOB: Drafting the article. SHK, NOB, YG, AEG, and SS: Critical revision of the article.

\section{Conflict of Interest Disclosures}

The authors have no conflicts of interest.

\section{Ethical Statement}

The study protocol was reviewed and approved by the ethics committee of the Istinye University Human Research Ethical Board (10.04.2020/31).

\section{Funding}

None.

\section{References}

1. Sangeeta M, Parthiban R, Vinila BH. Ectopic adrenal rests in testıs - a case report. Int J Basic Appl Med Sci. 2014;4(1):42-4.

2. Zengin T, Saracoglu M, Karaslan S, Ozturk H. Adrenal ectopic tissue in spermatic cord coincidence with testicular spermatocytic seminoma; a short view to literature. Cystoscope. 2013;1:55-7.

3. Penezic L, Hauptman D, Jelasic D, Kastelan Z. Ectopic adrenal tissue: A rarity among adult patients. Eur Urol Suppl. 2015;14(6):e1297. doi:10.1016/S1569-9056(15)30334-1.

4. Resorlu B, Soygur T, Kankaya D. Ectopic adrenocortical tissue in the spermatic cord in association with testicular teratoma. Ankara Univ Tip Fak Mecm. 2007;60(2):93-4. doi.
org/10.1501/Tipfak_0000000230.

5. Mares AJ, Shkolnik A, Sacks M, Feuchtwanger MM. Aberrant (ectopic) adrenocortical tissue along the spermatic cord. J Pediatr Surg. 1980;15(3):289-92. doi: 10.1016/s00223468(80)80139-4.

6. Sullivan JG, Gohel M, Kinder RB. Ectopic adrenocortical tissue found at groin exploration in children: incidence in relation to diagnosis, age and sex. BJU Int. 2005;95(3):407-10. doi: 10.1111/j.1464-410X.2005.05310.x.

7. Senescende L, Bitolog PL, Auberger E, Zarzavadjian Le Bian A, Cesaretti M. Adrenal ectopy of adult groin region: a systematic review of an unexpected anatomopathologic diagnosis. Hernia. 2016;20(6):879-85. doi: 10.1007/s10029-016-15351.

8. Mari M, Cagnazzi E, Bollito E, Bellina M. Ectopic adrenal cortex tissue in the spermatic cord in association with testicular cancer. Urol Int. 2004;73(2):191-2. doi: 10.1159/000079705.

9. Floyd MS, Itam S, Nasir N, Weerasinghe SM, Irwin PP, Maddineni SB. Concomitant testicular seminoma and ectopic adrenal tissue of the cord in a 45-year-old male. Can Urol Assoc J. 2014;8(3-4):e176-8. doi: 10.5489/cuaj.1584.

10. Yasar L, Elin M, Kaya C, Özkan Y. A rare case of ectopic adrenal tissue in the left ovary in a patient with a granulosacell tumor of the right ovary. J Gynecol Surg. 2010;28(3):2203. doi:10.1089/gyn.2011.0055.

11. Khandar B, Dey S, Ray PS, Sarkar R, Bhattacharyya P. Ectopic paratubal adrenal cell rest associated with mucinous cystadenoma of ovary. J Clin Diagn Res. 2015:9(10):ED13ED14. doi:10.7860/JCDR/2015/15411.6638.

12. Sahin C, Taylan E, Akdemir A, Zekioglu O, Seyidova P, Ergenoglu AM. Ovarian serous cystadenoma with ectopic adrenal tissue in a 65-year-old patient: A case report. Int J Surg Case Rep. 2017;33:89-91. doi: 10.1016/j.ijscr.2017.02.045.

13. Usta $U$, Turkmen E, Aydin NE. Adrenal ectopia within the wall of an ovarian serous cystadenoma. Balkan Med J. 2006;23:958.

14. Kim, T, Cha D, Han K, Youm H, Hyon N. Ectopic adrenal tissue in right uterine adnexa: A case report. Obstet Gynecol Sci. 2008;51(12):1562-6.

15. Sisto JM, Lu FW, Geffner ME, Berman ML. Para-ovarian adrenal rest tumors: gynecologic manifestations of untreated congenital adrenal hyperplasia. Gynecol Endocrinol. 2018;34(8):644-6. doi: 10.1080/09513590.2018.1441399.

16. Oshugi H, Takizawa N, Kinoshita H, Matsuda T. Pheochromocytoma arising from an ectopic adrenal tissue in multiple endocrine neoplasia type 2A. Endocrinol Diabetes Metab Case Rep. 2019;2019:19-0073. doi: 10.1530/EDM-190073.

17. Sugiyama T, Tajiri T, Hiraiwa S, Inomoto C, Kajiwara H, Kojima $S$, et al. Hepatic adrenal rest tumor: Diagnostic pitfall and proposed algorithms to prevent misdiagnosis as lipid-rich hepatocellular carcinoma. Pathol Int. 2015;65(2):95-9. doi: 10.1111/pin.12234.

18. Ye H, Yoon GS, Epstein JI. Intrarenal ectopic adrenal tissue and renal-adrenal fusion: a report of nine cases. Mod Pathol. 2009;22(2):175-81. doi: 10.1038/modpathol.2008.162.

19. Dotto RS, Marx G, Bastos M, Machado JL, GlufkeV, de Oliveira Freitas DM. A rare case of virilizing adult ectopic adrenal tumor. Urol Case Rep. 2019;27:100907. doi: 10.1016/j. eucr.2019.100907.

20. Mazza E, Papotti M, Durando R, Robecchi A, Camanni F. Ectopic aldosteronoma associated to another adrenocortical adenoma in the adrenal gland of the same side. J Endocrinol Invest. 1995;18(10):809-12. doi: 10.1007/BF03349816. 